\title{
Agent-based model for Johne's disease dynamics in a dairy herd
}

\author{
Jessica Robins², Sarah Bogen ${ }^{3}$, Auldon Francis², Annet Westhoek ${ }^{4}$, Andrew Kanarek', Suzanne Lenhart ${ }^{1}$ \\ and Shigetoshi Eda $2^{*}$
}

\begin{abstract}
Johne's disease is an infectious gastrointestinal disease in ruminants caused by Mycobacterium avium subsp. paratuberculosis that causes diarrhea, emaciation, decreased milk production and eventually death. The disease is transmitted in utero and via milk and colostrums to calves, and fecal-orally to all age classes. Financial losses due to the disease are estimated to be over $\$ 200$ million in the US dairy industry. The goal of this study was to evaluate the cost effectiveness of control measures based on diagnosis with a sensitive ELISA, EVELISA. An agent-based, discrete time model was developed to simulate Johne's disease dynamics in a US dairy herd. Spatial aspects of disease transmission were taken into account by using six spatial compartments. The effects on disease prevalence were studied with and without transmission routes included in the model. Further, using the model, cost effectiveness of ELISA-based Johne's disease control was evaluated. Using the parameters we collected and assumed, our model showed the initial prevalence of Johne's disease $(33.1 \pm 0.2 \%)$ in the farm increased to $87.7 \pm 1.7 \%$ in a 10 year-simulation. When ELISA-based control measures were included in the simulation, the increase in prevalence was significantly slowed down, especially when EVELISA was used. However, the level of the prevalence was still higher than the initial level after 10 year simulation even with the ELISA-based diagnostic intervention. The prevalence was further reduced when quarterly ELISA testing was included. The cost analysis showed that the quarterly ELISA and EVELISA testing could bring $\$ 44.8$ and $\$ 51.5 /$ animal/year more revenues, respectively, to a dairy farm.
\end{abstract}

\section{Introduction}

Johne's disease (JD) is an intestinal infection caused by Mycobacterium avium subsp. paratuberculosis (MAP) $[1,2]$. The disease infects wild [3] and domestic ruminants, including the dairy cow $[1,2]$. Symptoms include diarrhea, weight loss, decreased productivity and eventually death [1,2]. Johne's disease has spread worldwide [1]. Of the US dairy herds, $68.1 \%$ are infected [4]. Financial losses due to the disease were estimated to be between $\$ 200$ million and $\$ 250$ million annually, in the US dairy industry alone [5]. Although still debated, the pathogen might play a role in pathogenesis of Crohn's disease in humans [6,7].

Transmission of JD can occur mainly in three different ways. Calves can become infected in utero and via MAPcontaminated milk or colostrums $[1,2]$. The other transmission route, affecting all age classes, is fecal-oral, since the pathogen is also shed in the feces of infected individuals [8].

\footnotetext{
* Correspondence: seda@utk.edu

${ }^{2}$ University of Tennessee Institute of Agriculture, 2431 Joe Johnson Drive, 274 Ellington Plant Science Bldg, Knoxville, TN 37996-4563, USA

Full list of author information is available at the end of the article
}

The disease causing pathogen can persist in the environment for more than one year [9]. Infectiousness of animals, for all modes of transmission, is thought to depend on the amount of bacteria shed into the environment [10]. However, it can vary from less than 2 to more than 10 years before clinical symptoms are visible [11]. During this period, shedding at different levels may occur [11]. Shedding levels have been categorized in low, medium and high shedders depending on the number of colonies obtained by fecal culture or $\mathrm{Ct}$ value obtained by quantitative polymerase chain reaction. For example, Whitlock et al. [12] used 10 and 50 colonies/tube to separate low, medium and high shedders. Smith et al. found an association of shedding levels (low and high) with milk production of the MAP-infected cattle [13].

A variety of tests for JD is available, all with advantages and disadvantages. Fecal culture is the most definitive; however, the test is costly and takes up to 16 weeks to perform [14]. Polymerase chain reaction (PCR) based methods are faster but are more expensive than fecal culture test [14]. Enzyme-linked immunosorbent assay (ELISA) tests measure 
specific antibodies in blood samples. These tests are easy to perform, results are available within a week and the cost is less than one third of the cost for fecal culture [14]. However, the sensitivity relative to fecal culture is low, especially for low-shedding animals [12]. In 2006, Eda et al. [15] reported that sensitivity of ELISA test for JD can be improved by using ethanol extract of MAP and named the new ELISA, ethanol vortex ELISA (EVELISA).

Several management strategies to control JD are recommended. To decrease transmission fecal-orally, strategies include manure management to prevent contamination of feed and water, tilling contaminated pastures and general hygiene [14]. Calves require extra attention because of their higher susceptibility $[10,16]$ than adults. Strategies to prevent transmission via milk or colostrums include not pooling colostrums, only using colostrums from test-negative cows and feeding calves with pasteurized milk [14]. Culling test-positive animals is also recommended as a control strategy [14]. In that case, decisions to cull depend on the test used. Through modeling, we investigate the effects of various transmission routes on prevalence and economic outcomes of some testing regimes.

To analyze cost-effectiveness of ELISA-based JD control measures, an agent-based, discrete time model was developed to simulate JD dynamics in a dairy herd. The model incorporates contact structure, stochastic variation in demographic rates and disease dynamics. Two ELISAs with different sensitivities (i.e. current ELISA and EVELISA) were compared for their cost effectiveness. Our model includes some realistic features that were not included in two models $[17,18]$ which considered the cost-effectiveness of testing regimes. Details of the comparison of these two models with our model will be given in the discussion section.

In the next section, we describe our model in detail. The third section gives our numerical results showing the prevalence changes over time and economic analysis. We finish with a discussion of our results.

\section{Materials and methods}

\section{Model description}

A discrete time, stochastic, agent-based model was created and implemented using NetLogo. We describe the model construction using the standardized overview, design concepts, and details (ODD) protocol [19].

\section{Purpose}

The model was created to simulate JD and population dynamics in a dairy herd. The model may be used to provide insight into the importance of various routes of transmission and to investigate the epidemiological effects and economic impact of different diagnostic tests and management strategies.

\section{Entities, state variables, and scales}

Agents are defined as cows and represent individual cows on a dairy farm. As shown in Table 1, cows have the integer state variables of age, days since a strong positive diagnostic test, and days since a weak positive diagnostic test. These three state variables are reported in terms of days. Cows also have several binary state variables that allow them to behave collectively in a number of different contexts. The values of different Boolean state variables may indicate age group, disease status, location, or pregnancy status.

All state variables have the ability to change over time. State variables from the age group, location, and pregnancy categories only vary based on the progression of time. Changes in state variables from the disease status and diagnostic testing categories along with the addition and removal of cows include elements of stochasticity.

Each time step represents 1 day, and the model is run for up to 3650 days for population dynamics and for economic analysis. The model assumes random mixing of cows within a specific location which is a feature of each cow's current status. As shown in Figure 1, the location includes calf hatches, heifer group pen, pasture, pregnancy group pen, maternity barn and lactation barn.

\section{Process overview and scheduling}

During each time step, cows execute a specific sequence of processes. The first process is "grow", which includes aging and the possibility of giving birth. The second is "have-chance-of-infection", the third is "progress-indisease", and the fourth is "survive". The fifth is "move", which also incorporates diagnostic testing practices. All cows execute one of the five processes (or steps) before the entire population moves on to the next process in

Table 1 List of state variables used in the agent-based model for JD

\begin{tabular}{|c|c|c|c|c|c|}
\hline Category & Age group & Disease status & Location & Pregnancy & Diagnostic testing \\
\hline \multirow[t]{6}{*}{ State variables } & calf & healthy & Calf-hutch & Pregnant & Tested-strong \\
\hline & heifer & exposed & Heifer-group & & Tested-weak \\
\hline & adult & high-shedder & Pasture & & Days-after-strong \\
\hline & & low-shedder & Pregnancy-group & & Days-after-weak \\
\hline & & & Maternity-barn & & Weak-positive \\
\hline & & & Lactation-barn & & \\
\hline
\end{tabular}




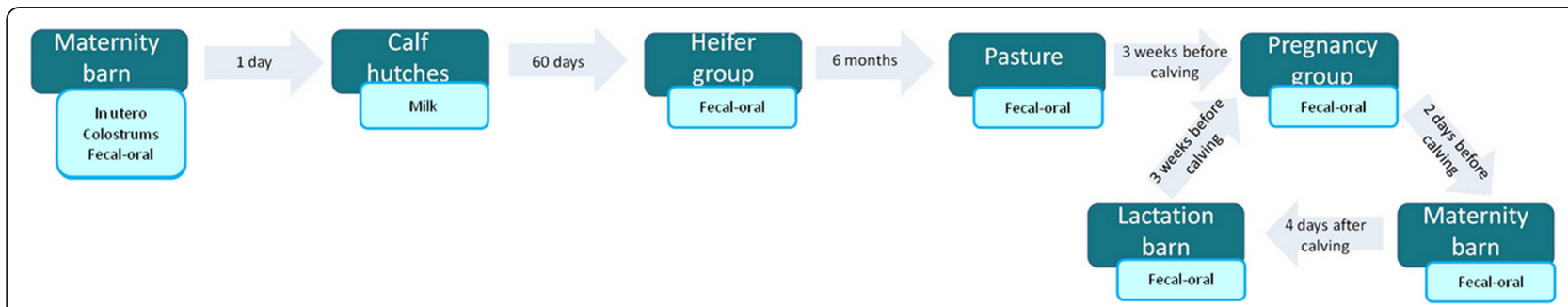

Figure 1 The scheme by which individuals move through different spatial compartments. Modes of disease transmission that occur in each compartment are indicated. Green squares indicate location of animals.

the sequence. Cows complete each process in a randomized turn-taking order. Any updates made to a particular cow's state variables as it is executing a process are implemented immediately. Selling and buying of heifers occur once a week and twice a year, respectively, to keep the population size consistent.

\section{Design concepts}

Basic principles The underlying design of the model is based largely on the common dairy farm management practice of grouping cows by age and providing separate housing for each group. The features of these separate housing environments vary based on the particular needs or characteristics of the individuals in each group. Due to the age differences and spatial variations in the environment for each group, different housing environments are likely to have different risks for transmission and routes of infection. The separation into six spatial compartments allows for these differences to be incorporated into the model. The spatial compartments, routes of disease transmission, and scheme for timebased movement between compartments used in the model are represented in Figure 1.

The separate spatial compartments allow for different routes of disease transmission in different age groups and spatial areas to be varied and tested independently. This helps provide insight into which age-specific or area-specific management practices would be most effective.

The structure of the diagnostic testing and actions taken due to test results are based on common and recommended practices. Individuals may test as strong positive, weak positive, or negative for JD either by ELISA or EVELISA. Individuals identified as strong positives are removed from the herd, and individuals identified as weak positives are tagged as such and their colostrums are not used to feed calves. These actions do not take place until 7 days pass in order to account for time between testing and obtaining results.

Emergence JD status of each animal is defined as susceptible (uninfected), exposed, low-shedding and highshedding. In this study, the term, "exposed", means that an animal is infected with MAP but not shedding MAP in its feces, milk and/or colostrums. The presence of each low shedding or high shedding individual contributes to the infection of other individuals in its respective spatial compartment via fecal-oral transmission, and thus also affects the change in prevalence indirectly. The dynamics of the prevalence values over time are expected to change when different disease transmission routes in different spatial compartments are turned on, turned off, or changed. They are also expected to change when certain management practices are applied to the entire herd, such as a testing and culling strategy.

Interaction Results of interactions between individuals are assumed based on different routes of disease transmission. Individuals born into the herd interact directly with their mothers during "chance of in-utero" infection and when drinking colostrums. New calves may also directly drink colostrums from one other mother in the maternity barn. Individuals are assumed to interact indirectly through fecal-oral transmission. Low shedding and high shedding individuals contribute to the environment within their respective spatial compartments, and the environment is assumed to contribute to the probability of infection of uninfected individuals in that spatial compartment.

Collectives Animals (agents) are grouped into collectives in three different ways: age class, state of disease, and spatial compartment. The age classes are calf, heifer, and adult. We assume that calves become heifers after weaning and that heifers become adults after giving birth for the first time. Animals in the calf group have ages 0-60 days, animals in the heifer group have ages $61-730$ days, and animals in the adult group have ages 731 days and above. Age classes are important when creating initial age distributions. States of disease include exposed, low shedding, and high shedding. Different disease states influence the infection dynamics of the entire population. The assigned spatial compartment is based on age and modeled after common dairy management practices. Locations include the maternity barn, the calf hutches, heifer group housing, the pasture, pregnancy group housing, and the 
lactation barn. Each compartment has its own set of transmission rates based on agents present in the group.

Stochasticity The processes of age initialization, disease initialization, successful female birth, natural mortality, disease transmission, disease progression, and diagnostic test results are all assumed to be stochastic. The stochasticity in age and disease initialization produces variability in the initial conditions. The purpose of the stochasticity in successful female birth, natural mortality, disease dynamics, and diagnostic test results is to replicate real-world frequencies of events.

Observation The prevalence of the disease in each stage and the number of individuals in each age class are tracked at each time step. Running totals of number of cows sold, number of cows bought, number of diagnostic tests administered, and number of cows culled are kept.

\section{Initialization}

During the model setup, cows are created and randomly assigned ages and infection status within a predetermined age structure. Of the initial cows, $10 \%$ are designated as calves, $40 \%$ are designated as heifers, and 50\% are designated as adults. Each calf is then assigned a random age between zero and sixty, each heifer a random age between 61 and 730, and each adult a random age between 731 and 2190. Each cow is then placed in the appropriate spatial compartment according to Table 2.

The numbers of each group are calculated based on the initial population, and members of each age group are randomly selected to change their infection status. Initial prevalence of JD used in this study is shown in Table 3.

\section{Input data}

The model does not use input data to represent timevarying processes.

\section{Functions (submodels)}

The Grow function advances each animal's age by 1 day. Depending on the new age, the animal may move to a new location (heifer group pen, maternity barn etc. shown in Figure 1), new age category (i.e. heifer or adult), and/or give a birth. If an animal gives a birth, its offspring has a $50 \%$ of chance to be female and has a chance to be infected (in utero or through colostrums).
The parameters used for these routes of infection are shown in Additional file 1. If the calf does not become exposed, it remains healthy. The new calf is then placed in the maternity barn.

The have-chance-of-infection function implements infection of healthy calves through colostrums from a second mother and fecal-oral transmission of all healthy cows.

If a calf is healthy newborn in the maternity barn and colostrums from another dam is set to be on, it has a $50 \%$ chance of drinking from any non-pregnant adult animal in the maternity barn. One dam is selected at random. If the dam selected is a low shedder and it has not been classified as a weak positive through ELISA or EVELISA testing, the calf has a chance of becoming exposed. Similarly, if the dam selected is a high shedder and has not been classified as a weak positive through ELISA or EVELISA testing, the calf has a higher chance of becoming exposed than the case the dam is a low shedder. Also, calves can become infected through drinking milk from low and high shedders. Fecal-oral transmission occurs independently in each compartment. This probability of fecal-oral transmission is defined by the following function:

$$
\text { infection chance }_{\text {comp }}=\beta \frac{(1-\gamma) \text { low shedders }_{\text {comp }}+(\gamma) \text { high shedders }}{\text { comp }}
$$

Where, the "infection chance comp" is the probability of an animal in the compartment getting infected in 1 day. The subscript "comp" indicates the compartment where the animal locates on that day. low-shedders comp $_{\text {, high- }}$ shedder $_{\text {comp }}$ and total-population comp are the number of animals in the compartment on that day. $\beta$ is the transition rate (set to $0.002,0.0002$, and 0.00002 for calves, heifers and adults, respectively) from susceptible (uninfected) animals to exposed (infected but not shedding) animals. Parameter $\gamma$, which is set to 0.9 for the entire study, allows for high shedders to impact infection transmission more than low shedders. Fecal-oral route infections occur in all the compartments except for calf hatches where calves are well separated.

In the Progress-in-disease (transition from exposed to low shedder and then to high shedder) function, each low shedder has a probability of becoming a high shedder and each exposed cow has an exposed-to low chance of becoming a low shedder.

Table 2 Days for translocation of animals

\begin{tabular}{lllllll}
\hline Spatial compartment & Calf hutches & $\begin{array}{l}\text { Heifer group } \\
\text { housing }\end{array}$ & Pasture & $\begin{array}{l}\text { Pregnancy group } \\
\text { housing }\end{array}$ & Maternity barn & Lactation barn \\
\hline Age & $0-60$ & $61-179$ & $180-710$ & $711-728,1066-1124$, & $729-735,1125-1131$, & $736-1065,1132-1461$, \\
& & & & $1462-1520,1858-1916$ & $1521-1527,1917-1923$ & $1528-1859,1923-2190$ \\
\hline
\end{tabular}


Table 3 Initial JD prevalence

\begin{tabular}{llll}
\hline & Exposed & Low-shedding & High-shedding \\
\hline Calves & $35 \%$ & $0 \%$ & $0 \%$ \\
Heifers & $31 \%$ & $4 \%$ & $0 \%$ \\
Adults & $25 \%$ & $8 \%$ & $2 \%$ \\
\hline
\end{tabular}

The Survive (natural mortality rates) function simulates removal from the farm due to natural death or timely removal. There are separate daily survival rates for calves in their first $48 \mathrm{~h}$ of life, other calves, heifers, and adults. The survival rate for the first $48 \mathrm{~h}$ includes unsuccessful births, and the adult survival rate includes removal due to old age or disease. Cows that are not successful in the survive function are removed from the population.

During the Move function, each cow may be relocated to the appropriate new compartment based on its new age assigned in Grow.

The Test function implements ELISA (or EVELISA) testing. The model allows for the use of ELISA testing, EVELISA testing, or neither test, with multiple testing up to four times a year. A cow may test as a strong positive, a weak positive, or a negative and are tagged as such. Details are described in our previous paper [17]. There are different probabilities for each test result depending on the fecal shedding status of the animal (i.e. no-shedding (susceptible or exposed), low shedding, or high shedding). Seven days (time required to get test results back) after the testing, cows that tested as strong positives are removed from the herd and those that tested as weak positives are tagged as known weak positives. Cows that are classified as known weak positives do not contribute colostrums toward feeding calves.

The Buy function occurs every buying-interval (182 days) when the total number of cows is less than the initial population. Cows are introduced until the total number of cows is equal to the initial population. New cows are springing heifers and are placed the pregnancy group housing. These new cows are initialized as healthy, exposed or low shedding according to the initial prevalence which was determined based on the regional prevalence.

The Sell function acts every 7 days if the total number of cows is greater than the initial population. Cows classified as heifers are removed from the population until the total number of cows is equal to the initial population.

\section{Simulations}

Simulations were run to provide insight into the relative importance of each route of disease transmission, the effect of eliminating fecal-oral transmission in the pasture, and the epidemiological and economic effects of employing a test and cull strategy using the ELISA test or the EVELISA test. Due to the stochastic nature of the model, ten runs were simulated for each parameter combination. Results were measured at each time step in terms of prevalence of exposed cows, prevalence of low shedding cows, prevalence of high shedding cows, and total prevalence. For the simulations measuring economic impact, results were reported in terms of net gain or net loss at the end of each iteration.

Tested scenarios are as follows:

1) Contribution of each transmission pathway (no ELISA-based intervention).

- All transmission pathways possible

- No fecal-oral transmission

- No transmission through MAP contaminated milk

- No transmission through contaminated colostrums

- No in utero transmission

2) Impact of ELISA-based interventions (all transmission pathways possible)

- No testing

- ELISA test-based interventions (once or four times a year)

- EVELISA test-based interventions (once or four times a year).

\section{Economic analysis}

The revenue was calculated to be (sales of milk and culled cows) minus (payments for replacement heifers and testing). Other costs are assumed to be consistent in each scenario and whereas not included in the calculation.

\section{Results}

Population dynamics of JD in a dairy farm was simulated using the agent-based model developed in this study. When all the transmission routes were included, the total prevalence of JD (total infected animals, exposed + low shedders + high shedders) increased from the initial level (average \pm standard deviation: $33.1 \pm 0.2 \%$ ), to $87.7 \pm$ $1.7 \%$ in the 10 year simulation (Figure 2A). The prevalence of JD in each age group at the end of the 10-year simulation was $21.9 \pm 5.4 \%, 32.9 \pm 1.6 \%$ and $41.5 \pm 2.6 \%$ for calves, heifer and adults, respectively. When fecal-oral route transmission was removed from the simulation, the total prevalence did not increase but persisted (Figure 2B). Removal of transmissions through milk (Figure 2C), colostrums (Figure 2D) and vertical transmission (Figure 2E) had much less effect on the increase in prevalence and the total prevalence at the end of the simulations were about $71.6 \pm 1.8 \%, 77.3 \pm 1.9 \%$ and $86.1 \pm 1.6 \%$, respectively.

In this study, assumed values were used for infection rate for each transmission route (i.e. fecal-oral, milk, 


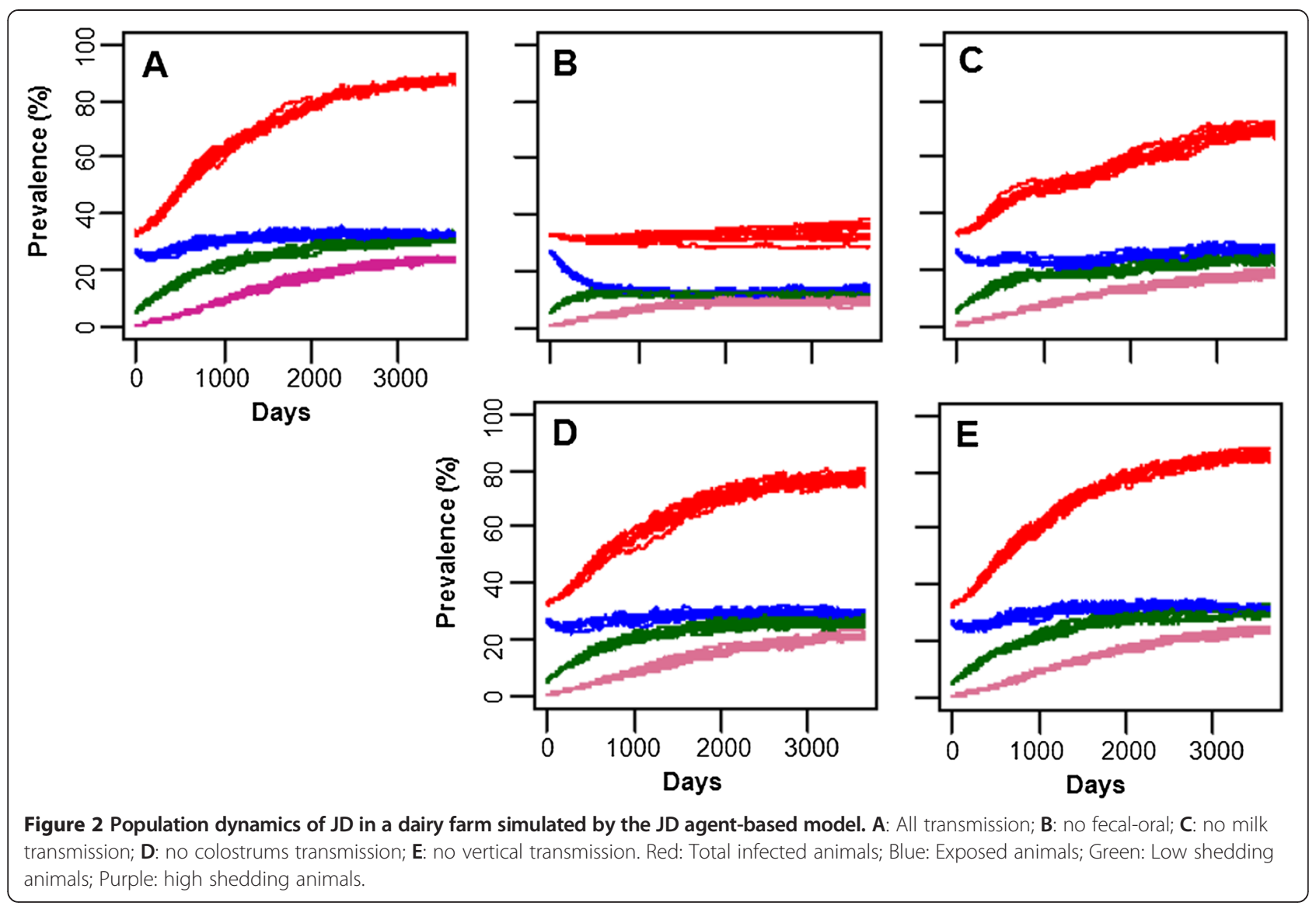

colostrums and vertical) as listed in Additional file 1. To evaluate influence of each assumed parameter on prevalence, simulations were run with halved or doubled level of the parameter. Influence on prevalence was evaluated by finding number of days that required for the total prevalence to reach $50 \%$. As shown in Figure 3, infection rate for fecal-oral transmission route, followed by that for milk transmission route, had the most significant influence on rate of prevalence increase. Changing infection rates for colostrums and vertical transmission routes did not result in any statistically significant difference. With all the transmission modes included, ELISAor EVELISA-based control measures were applied in the model (Figure 4). When results of ELISA and EVELISA testing (once a year) was used to control JD in the dairy farm, the prevalence after 10 year simulation was reduced to $50.9 \pm 1.6 \%$ and $36.2 \pm 1.6 \%$, respectively (Figures $4 \mathrm{~B}$ and $\mathrm{C}$ ). As shown in Figures $4 \mathrm{D}$ and $\mathrm{E}$, more significant reductions (ELISA: $28.4 \pm 3.5 \%$ and EVELISA: $15.7 \pm 1.9 \%$ ) was observed when the testing was conducted quarterly (four times a year).

Using recent values of milk, replacement heifer, culled cow and ELISA testing, revenues of the simulated dairy farm was calculated under different scenarios of JD control. With annual testing, ELISA- and EVELISAbased control measures reduced revenue of the simulated dairy farm and levels of the reduction were greater for EVELISA (Table 4). When testing frequency was increased to four times a year, ELISA- and EVELISA-based control showed higher revenues than no-testing scenario at the initial prevalence of 10 and 20\%; however, the difference was not statistically significant. Statistically significant differences were observed for ELISA- and EVELISA-based controls when the simulations were run for 20 years. The increases in revenue are 1.79 and 2.06 million US dollars/ 20 years for ELISA and EVELISA, respectively.

\section{Discussion}

Epidemiological studies of MAP have been hampered by the fact that currently-used diagnostic tests are incapable of detecting the early (latent) stage of MAP infections. For better understanding of JD epidemiology, mathematical modeling approach has been employed since the early '90s. Collins et al. [20] presented the first mathematical model describing the behavior of JD in an open herd. For evaluation of JD management strategies, Groenendaal et al. [21] developed stochastic models-named "JohneSSim". Since 2008, 


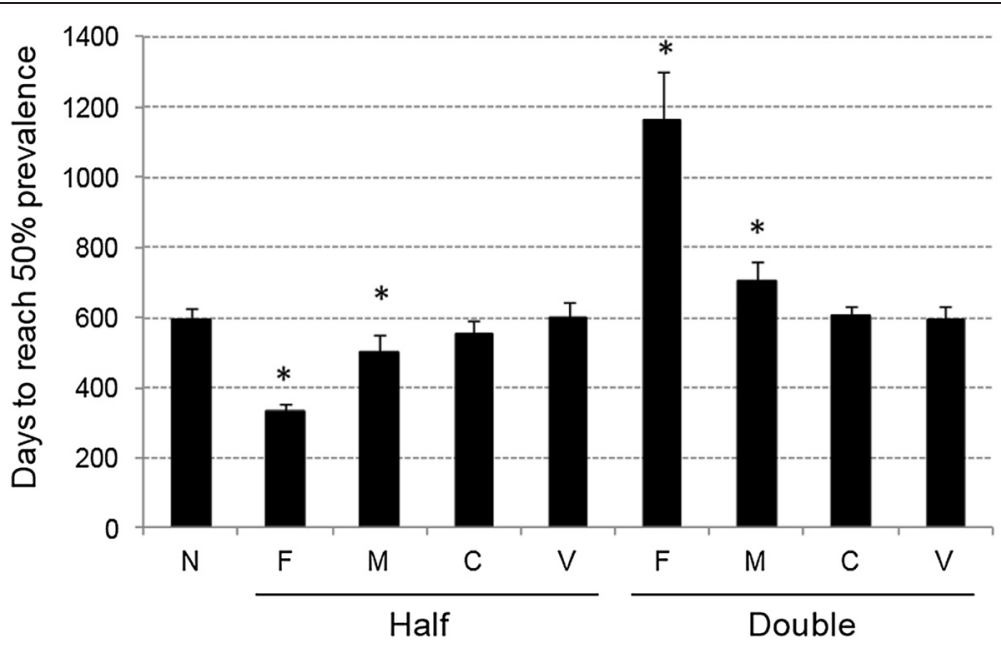

Figure 3 Influence of assumed parameters on transmission of MAP. In this study, assumed values were used for infection rates for fecal-oral (F), milk (M), C (colostrums) and V (vertical) transmissions. Simulations were run with halved or doubled each infection rate. Each bar represents days that required for the total prevalence (exposed + low shedding + high shedding animals) to reach $50 \%$. The error bars indicate standard deviation of data obtained by 10 simulations. Statistical significance among the group was detected by ANOVA test. Asterisks indicate that a statistical significant between the data and the original data $(\mathrm{N})$ was detected by pair-wise t- test with Bonferroni and Holm adjustments.

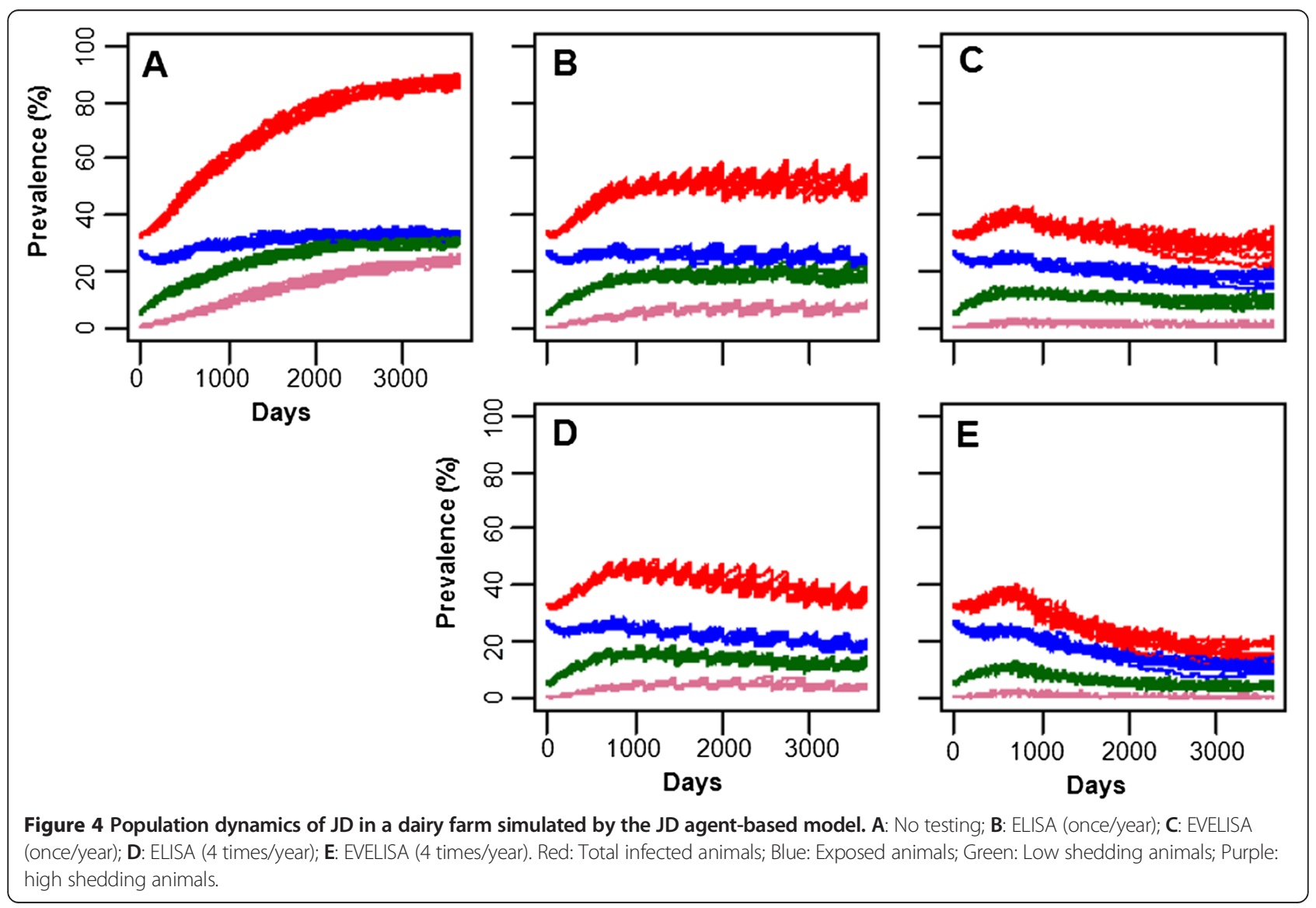


Table 4 Economic analysis of ELISA-based control measures

\begin{tabular}{|c|c|c|c|c|c|}
\hline Testing & $\begin{array}{l}\text { Initial } \\
\text { prevalence (\%) }\end{array}$ & Control & Average $^{b}$ & SD & Difference \\
\hline \multirow[t]{9}{*}{ Once } & 5 & None & 32.4 & 0.22 & \\
\hline & & ELISA & 32.0 & 0.08 & $-0.38^{c}$ \\
\hline & & EVELISA & 31.9 & 0.18 & $-0.44^{c}$ \\
\hline & 10 & None & 31.9 & 0.14 & \\
\hline & & ELISA & 31.4 & 0.14 & $-0.53^{c}$ \\
\hline & & EVELISA & 31.3 & 0.19 & $-0.61^{c}$ \\
\hline & 20 & None & 31.5 & 0.18 & \\
\hline & & ELISA & 31.0 & 0.18 & $-0.45^{c}$ \\
\hline & & EVELISA & 30.8 & 0.23 & $-0.64^{c}$ \\
\hline \multirow[t]{9}{*}{ Four times } & 5 & None & 32.4 & 0.22 & \\
\hline & & ELISA & 32.2 & 0.18 & -0.13 \\
\hline & & EVELISA & 32.2 & 0.17 & -0.18 \\
\hline & 10 & None & 31.9 & 0.14 & \\
\hline & & ELISA & 32.0 & 0.16 & 0.01 \\
\hline & & EVELISA & 32.0 & 0.11 & 0.07 \\
\hline & 20 & None & 31.5 & 0.18 & \\
\hline & & ELISA & 31.6 & 0.14 & 0.09 \\
\hline & & EVELISA & 31.6 & 0.18 & 0.17 \\
\hline
\end{tabular}

aPrevalence of shedders in adult population. The unit of the monetary numbers is million dollars $/ 10$ years ${ }^{a}$. Ten percent is the same distribution of prevalence shown in Table 3 ; $^{\mathrm{b}}$ Average revenue calculated from results of 10 simulations; and 'statistically different (ANOVA followed by $t$ test with Bonferroni and Holm adjustments, $p<0.01$ ) from no ELISA-based control.

Mitchell et al. [22] and $\mathrm{Lu}$ et al. [23,24] developed mathematical models that incorporated a "transient shedding" within the calf population. Lu et al. [24] employed a stochastic compartmental model to better evaluate fadeout of JD in dairy herds. These works are reviewed in a recent publication [25].

In these previous models, there were some missing factors that would be important for understanding of JD epidemiology. For example, most of these models assumed that animals become resistant to MAP infection after one year of age; however, some findings $[16,26,27]$ indicated that adult animals could also get infected with MAP and developed JD. Also, the contact structure in a dairy herd was not incorporated in mathematical models of JD with exception of a recent report [28]. Further, only a limited number of studies employed agent-based modeling approach. We therefore elected to develop an agent-based model incorporating MAP infection in adult animal population and contact structure. Agent-based model captures emergent phenomena, provides a natural description of the modeled system and is flexible especially in geospatial models.

Our model predicted that, if no control measure was applied, the initial prevalence of JD in the modeled herd 33.1 $\pm 0.2 \%)$ would increased to $87.7 \pm 1.7 \%$ after a 10 year- simulation, which is similar to the prediction obtained by a previous modeling work [18]. The end-point prevalence of $90 \%$ may sound very high but is possible because the prevalence include exposed (latent) animals whose number was reported in this special issue to be 2.5 times higher than that of fecal culture positive animals [29] and the prevalence of fecal culture positive animals could reach close to $40 \%$ [30].

Similar to the model presented in this study, our previous model [17] was developed using a contact structure in a dairy herd but was based on a set of difference equations. The previous model predicted that use of EVELISA was more cost effective (40 US dollars/cow/ 10 years) than the current ELISA test. The most closely related work to this study was conducted by Kudahl et al. in 2007 [18]. Their model is based on SimHerd which is an agent-based model but, in contrast to our study, contact structure was not considered. Another major difference is that our study used sensitivities of commercial ELISA and EVELISA obtained by testing a same set of filed samples whereas the previous study used assumed values for the ELISA with a higher sensitivity (improved ELISA). Their model predicted that, after 10 years of ELISA-based control, the improved ELISA is more cost-effective (70-80 Euro/cow/10 years) than current ELISA when initial prevalence was set to $25 \%$ and test- $\&$-cull control strategy (quarterly for $<4$ years old animals and annually for older animals) was implemented in their model. In our study, EVELISA was predicted to be more cost-effective (67.5US dollars/cow/10 years) than the current ELISA when initial fecal culture positive prevalence was set to $10 \%$ and quarterly test-\&-cull was implemented for 20 years in the model.

Currently, ELISA testing for JD control is conducted only once a year [31]. Our model predicted that even though the increase in JD prevalence could be slowed down by applying annual ELISA-based control, there would be a negative impact on revenue. Although the quarterly test-\&-cull control was able to significantly reduce the prevalence and also predicted to be costeffective, it will increase labor for testing and is currently not realistic. Recent work has demonstrated that an onsite diagnostic device for JD could be developed by using a capacitance sensing approach [32], and once fully developed, such a device would make it easier and cheaper to implement a quarterly test and cull procedure.

\section{Additional file}

Additional file 1: List of parameters used in this study. Parameter

values and their source were listed $[4,12-14,17,18,23,30,33,34]$.

\section{Abbreviations}

JD: Johne's disease; MAP: Mycobacterium avium subsp. paratuberculosis; ELISA: Enzyme-linked immunosorbent assay; EVELISA: Ethanol-vortex ELISA; PCR: Polymerase chain reaction; ODD: Overview, design concepts, and details. 


\section{Competing interests}

An EVELISA-based ELISA kit was commercialized and SE has a right to receive royalties.

\section{Authors' contributions}

SL and SE designed and organized the study. JR, SB, AF, and AW developed the model, ran the analysis, and prepared this manuscript under supervision of SL and SE. AK helped to refine the model. All authors read and approved the final manuscript.

\section{Acknowledgements}

We thank Drs. Don Klinkenberg, Yrjö Gröhn and Vitaly Ganusov for careful review and productive comments on this manuscript. The authors acknowledge the support of the Within-host modeling of MAP infections Working Group at the National Institute for Mathematical and Biological Synthesis, sponsored by the National Science Foundation, the U.S. Department of Homeland Security, and the U.S. Department of Agriculture through NSF Award DBI-1300426, with additional support from The University of Tennessee, Knoxville.

\section{Author details}

${ }^{1}$ National Institute for Mathematical and Biological Synthesis, University of Tennessee Knoxville, 1122 Volunteer Blvd., Suite 106, Knoxville, TN 37996-3410, USA. ${ }^{2}$ University of Tennessee Institute of Agriculture, 2431 Joe Johnson Drive, 274 Ellington Plant Science Bldg, Knoxville, TN 37996-4563, USA. ${ }^{3}$ Capital University, 1 College Ave Bexley, OH, USA. ${ }^{4}$ Wageningen University, 6708PB, Wageningen, The Netherlands.

\section{Received: 15 September 2014 Accepted: 5 March 2015}

\section{Published online: 19 June 2015}

\section{References}

1. Stabel JR (1998) Johne's disease: a hidden threat. J Dairy Sci 81:283-288

2. Sweeney RW, Collins MT, Koets AP, McGuirk SM, Roussel AJ (2012) Paratuberculosis (Johne's disease) in cattle and other susceptible species. J Vet Intern Med 26:1239-1250

3. Raizman EA, Wells SJ, Jordan PA, DelGiudice GD, Bey RR (2005) Mycobacterium avium subsp. paratuberculosis from free-ranging deer and rabbits surrounding Minnesota dairy herds. Can J Vet Res 69:32-38

4. USDA/NAHMS report, Johne's Disease on U.S. Dairies, 1991-2007. http://www.aphis.usda.gov/animal_health/nahms/dairy/downloads/dairy07/ Dairy07_is_Johnes.pdf, Accessed 7 May 2015

5. Ott SL, Wells SJ, Wagner BA (1999) Herd-level economic losses associated with Johne's disease on US dairy operations. Prev Vet Med 40:179-192

6. Naser SA, Sagramsingh SR, Naser AS, Thanigachalam S (2014) Mycobacterium avium subspecies paratuberculosis causes Crohn's disease in some inflammatory bowel disease patients. World J Gastroenterol 20:7403-7415

7. Chiodini RJ, Chamberlin WM, Sarosiek J, McCallum RW (2012) Crohn's disease and the mycobacterioses: a quarter century later. Causation or simple association? Crit Rev Microbiol 38:52-93

8. Aly SS, Thurmond MC (2005) Evaluation of Mycobacterium avium subsp paratuberculosis infection of dairy cows attributable to infection status of the dam. J Am Vet Med Assoc 227:450-454

9. Elliott GN, Hough RL, Avery LM, Maltin CA, Campbell CD. Environmental risk factors in the incidence of Johne's disease. Crit Rev Microbiol (in press)

10. Mitchell RM, Medley GF, Collins MT, Schukken YH (2012) A meta-analysis of the effect of dose and age at exposure on shedding of Mycobacterium avium subspecies paratuberculosis (MAP) in experimentally infected calves and cows. Epidemiol Infect 140:231-246

11. Nielsen SS, Ersboll AK (2006) Age at occurrence of Mycobacterium avium subspecies paratuberculosis in naturally infected dairy cows. J Dairy Sci 89:4557-4566

12. Whitlock RH, Wells SJ, Sweeney RW, Van Tiem J (2000) ELISA and fecal culture for paratuberculosis (Johne's disease): sensitivity and specificity of each method. Vet Microbiol 77:387-398

13. Smith RL, Grohn YT, Pradhan AK, Whitlock RH, Van Kessel JS, Smith JM, Wolfgang DR, Schukken YH (2009) A longitudinal study on the impact of Johne's disease status on milk production in individual cows. J Dairy Sci 92:2653-2661
14. Collins MT, Gardner IA, Garry FB, Roussel AJ, Wells SJ (2006) Consensus recommendations on diagnostic testing for the detection of paratuberculosis in cattle in the United States. J Am Vet Med Assoc 229:1912-1919

15. Eda S, Bannantine JP, Waters WR, Mori Y, Whitlock RH, Scott MC, Speer CA (2006) A highly sensitive and subspecies-specific surface antigen enzyme- linked immunosorbent assay for diagnosis of Johne's disease. Clin Vaccine Immunol 13:837-844

16. Windsor PA, Whittington RJ (2010) Evidence for age susceptibility of cattle to Johne's disease. Vet J 184:37-44

17. Massaro T, Lenhart S, Spence M, Drakes C, Yang G, Agusto F, Johnson R, Whitlock B, Wadhwa A, Eda S (2013) Modeling for cost analysis of Johne's disease control based on EVELISA testing. J Biol Syst 21:1340010

18. Kudahl AB, Sorensen JT, Nielsen SS, Ostergaard S (2007) Simulated economic effects of improving the sensitivity of a diagnostic test in paratuberculosis control. Prev Vet Med 78:118-129

19. Grimm V, Berger U, DeAngelis DL, Polhill JG, Giske J, Railsback SF (2010) The ODD protocol A review and first update. Ecol Model 221:2760-2768

20. Collins MT, Morgan IR (1991) Epidemiological model of paratuberculosis in dairy cattle. Prev Vet Med 11:131-146

21. Groenendaal $H$, Nielen $M$, Jalvingh AW, Horst SH, Galligan DT, Hesselink JW (2002) A simulation of Johne's disease control. Prev Vet Med 54:225-245

22. Mitchell RM, Whitlock RH, Stehman SM, Benedictus A, Chapagain PP, Grohn YT, Schukken YH (2008) Simulation modeling to evaluate the persistence of Mycobacterium avium subsp. paratuberculosis (MAP) on commercial dairy farms in the United States. Prev Vet Med 83:360-380

23. Lu Z, Mitchell RM, Smith RL, Van Kessel JS, Chapagain PP, Schukken YH, Grohn YT (2008) The importance of culling in Johne's disease control. J Theor Biol 254:135-146

24. Lu Z, Schukken YH, Smith RL, Grohn YT (2010) Stochastic simulations of a multi-group compartmental model for Johne's disease on US dairy herds with test-based culling intervention. J Theor Biol 264:1190-1201

25. Marce C, Ezanno P, Weber MF, Seegers H, Pfeiffer DU, Fourichon C (2010) Invited review: modeling within-herd transmission of Mycobacterium avium subspecies paratuberculosis in dairy cattle: a review. J Dairy Sci 93:4455-4470

26. Wells SJ, Kubat N, Espejo LA, Godden SM. Effect of delaying exposure to Mycobacterium avium subsp. paratuberculosis until adulthood on incidence of infection in adult dairy cows. In 11th International Colloquium on Paratuberculosis; Sydney, Australia. 2012;332.

27. Espejo LA, Kubat N, Godden SM, Wells SJ (2013) Effect of delayed exposure of cattle to Mycobacterium avium subsp paratuberculosis on the development of subclinical and clinical Johne's disease. Am J Vet Res 74:1304-1310

28. Marce C, Ezanno P, Seegers H, Pfeiffer DU, Fourichon C (2011) Within-herd contact structure and transmission of Mycobacterium avium subspecies paratuberculosis in a persistently infected dairy cattle herd. Prev Vet Med 100:116-125

29. Schukken YHS, Mitchell RM, Whitlock RH, Grohn YT (2015) Longitudinal data collection of Mycobacterium avium subspecies paratuberculosis infections in dairy herds. Vet Res (in press)

30. Espejo LA, Godden S, Hartmann WL, Wells SJ (2012) Reduction in incidence of Johne's disease associated with implementation of a disease control program in Minnesota demonstration herds. J Dairy Sci 95:4141-4152

31. Geraghty T, Graham DA, Mullowney P, More SJ (2014) A review of bovine Johne's disease control activities in 6 endemically infected countries. Prev Vet Med 116:1-11

32. Li S, Cui H, Yuan Q, Wu J, Wadhwa A, Eda S, Jiang H (2014) AC electrokineticsenhanced capacitive immunosensor for point-of-care serodiagnosis of infectious diseases. Biosens Bioelectron 51:437-443

33. Gould BW (2006) Understanding Dainy Markets. University of Wisconsin, Madison

34. USDA-ERS. Milk production costs and returns per hundredweight sold, 2010-2011. 2012. 